\title{
Computational Modeling of Plasmon-Enhanced Light Absorption in a Multicomponent Dye Sensitized Solar Cell
}

\author{
Hanning Chen, Martin G. Blaber, Stacey D. Standridge, Erica J. DeMarco, Joseph T. Hupp, \\ Mark A. Ratner, and George C. Schatz*
}

Argonne-Northwestern Solar Energy Research Center Department of Chemistry, Northwestern University, 2145 Sheridan Road, Evanston, Illinois 60208, United States

\section{Supporting Information}

ABSTRACT: Plasmon-enhanced light absorption in a multicomponent $\mathrm{Ag} / \mathrm{Ag}_{2} \mathrm{O} / \mathrm{TiO}_{2} / \mathrm{N} 3$ dye-sensitized solar cell (DSSC) core-shell nanostructure is studied using a hybrid quantum mechanics/classical electrodynamics (QM/ED) methodology in which the $\mathrm{Ag} / \mathrm{Ag}_{2} \mathrm{O} / \mathrm{TiO}_{2}$ nanostructure is treated by the finite-difference time-domain method and the N3 dye is treated by real-time time-dependent density functional theory. As part of this modeling, the undetermined thickness of the nonplasmonic $\mathrm{Ag}_{2} \mathrm{O}$ layer on the $\mathrm{Ag} / \mathrm{Ag}_{2} \mathrm{O} / \mathrm{TiO}_{2}$ particle was estimated by comparing the computed plasmon wavelength with experimental results. Also, absorption cross sections for the $\mathrm{N} 3$ dye were calculated for different locations of the dye on the $\mathrm{TiO}_{2}$ surface. The spatially averaged absorption cross sections for different thicknesses of $\mathrm{TiO}_{2}$ were evaluated and used to estimate the relative incident photon conversion efficiency. Encouragingly, it is found that the QM/ED calculations can well reproduce the factor of $\sim 10$ experimental extinction difference spectrum and the photocurrent enhancement factor associated with DSSCs. Our studies demonstrate that the hybrid QM/ED methodology provides a useful guide to the systematic design of plasmon-enhanced DSSCs for achieving optimum photovoltaic efficiency.

\section{INTRODUCTION}

Solar cells are one of the most promising approaches to environmentally friendly and ecologically sustainable energy sources. ${ }^{1,2}$ Despite a half century of persistent efforts on improving photovoltaic efficiency, the wide deployment of the solar cell technology is still severely hindered by its expensive manufacturing cost and short product lifetime. ${ }^{3}$ Currently, most commercially available solar cell panels are made of monocrystalline silicon doped with toxic heavy metals, making the disposal and recycling of the panels at the end of their useful life a challenging task. Fortunately, both the economical and the environmental issues associated with semiconductor based solar cells can be mitigated by using dye-sensitized solar cells (DSSCs), ${ }^{4}$ which use organic dye molecules coated on $\mathrm{TiO}_{2}$ particles as the photoactive component to reduce fabrication expenses and eliminate expensive and toxic ingredients. In addition, the DSSC is known to function even under low-light conditions and to operate at low internal temperature, thanks to its ultrafast electron injection ${ }^{5}$ and its ultrathin conductive film, ${ }^{6}$ respectively. However, the relatively low photovoltaic efficiency of DSSCs $(\sim 10 \%)^{7}$ compared to crystalline silicon solar cells $(\sim 30 \%)^{8}$ is a major drawback. This disadvantage has been largely ascribed to the dye molecules' low light absorption due to low extinction coefficients, ${ }^{4}$ although the components in a DSSC are highly coupled such that just changing one factor is not sufficient to improve an optimized cell.

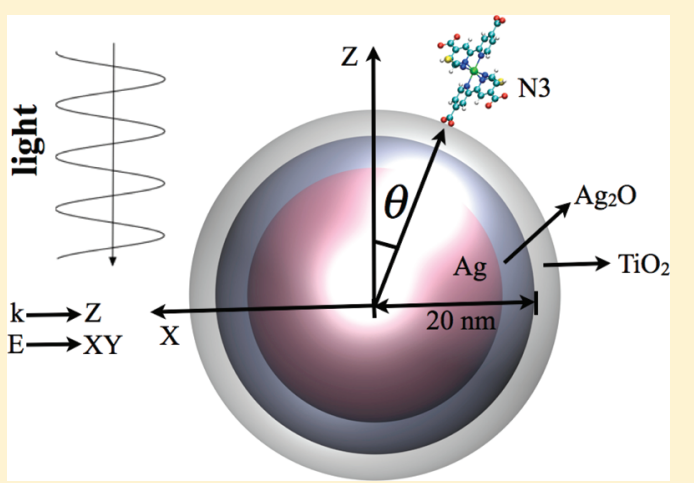

Multilayer fabrication strategies have been suggested for plasmonic DSSCs by anchoring the dye molecules on the surface of $\mathrm{TiO}_{2}$-coated plasmonic (silver or gold) particles. ${ }^{9,10}$ With one version of this cell, the monochromatic photocurrent of an optically thin $\mathrm{N} 3 / \mathrm{TiO}_{2} / \mathrm{Ag}$ composite DSSC was improved by nearly an order of magnitude through plasmon enhanced absorption by the dye. ${ }^{10}$ This particular solar cell was intentionally designed to be optically thin, and therefore, the overall cell efficiency even with plasmon enhancement was only $0.05 \%$, but this is a useful cell for studying mechanistic issues (and therefore we consider it in our analysis below). In particular, it was demonstrated that the photocurrent enhancement and absorption enhancement are very similar, indicating that the primary effect of the plasmonic particles is to enhance absorption. Also, the incident photon conversion efficiency (IPCE) of the cell was shown to be very sensitive to the thickness of the $\mathrm{TiO}_{2}$ layer located between the $\mathrm{N}_{3}$ pigments and the $\mathrm{Ag}$ nanoparticles, suggesting the possibility of improving the performance of plasmon-enhanced DSSC (PDSSC) through optimization of the interfacial nanoarchitectures.

The improvability of DSSC performance associated with plasmon excitation has been further demonstrated by a more

Received: February 28, 2012

Revised: April 10, 2012

Published: April 11, 2012 
recent study that exhibits a perfect match at $525 \mathrm{~nm}$ between the absorption maxima of the Z907 dye and the plasmon wavelength of $\mathrm{SiO}_{2}$-coated $\mathrm{Au}$ nanoparticles when both of them are integrated into mesoporous $\mathrm{TiO}_{2} \cdot{ }^{11}$ Although a shorter excited-state lifetime is usually observed when a dye molecule interacts with a plasmonic resonance, ${ }^{12}$ the overall rate of electron transfer is still faster. Presumably, this is due to plasmon-enhanced light absorption, which arises from the locally amplified field at the position of the dye molecules. ${ }^{13}$

In another recent study, Jeong et al. ${ }^{14}$ demonstrated a $25 \%$ improvement in efficiency with plasmon-enhancement for a PDSSC solar cell that has an overall efficiency of $8.9 \%$. Related results have been reported in other papers. ${ }^{15,16}$ These show that plasmon enhancement effects can be important even for cells that have high efficiency.

In order to understand fully the mechanism for plasmonenhanced light absorption by dye molecules on thin-film electrodes and to aid in the systematic design of P-DSSCs to achieve maximum IPCE results, a general theoretical model would be very useful. Such a model needs to describe the quantum characteristics of individual electronic transitions of the dye molecules; it also needs to describe the optical response of the coated metallic nanoparticles. Since the latter is mainly induced by the large collective motions of the conduction electrons, a classical electrodynamics mean-field approach should be sufficient. It has been shown that accurate simulations of the optical response of small particles and nanostructures with gaps on the order of $1 \mathrm{~nm}$ requires the inclusion of quantum effects in the electrodynamics. ${ }^{17,18}$ Here, the particles have diameters $>10 \mathrm{~nm}$, and as such, a classical electrodynamics mean-field approach should be adequate. Although quantum effects can be neglected from the electrodynamics of the nanoparticle, a unified theoretical framework is still required, balancing the accuracy of quantum theory for the molecule and the efficiency of classical electrodynamics for the particle. To meet these challenges, a hybrid quantum mechanics/classical electrodynamics (QM/ED) method ${ }^{19}$ was recently developed. This method dynamically incorporates polarization associated with the nanostructure into an optical property calculation for nearby dye molecules. Our version of $\mathrm{QM} / \mathrm{ED}$ is done in the time-domain, which means that the dipole coupling Hamiltonian is added to the dye molecule's time-dependent quantum Hamiltonian to determine the induced polarization in the molecule, from which the optical behavior is determined. The time-dependent electric field in this dipole Hamiltonian is described by classical electrodynamics associated with light interacting with the nanoparticle, and in this case, we obtain the time-dependent field using the finite-difference time-domain (FDTD) method. The hybrid $\mathrm{QM} / \mathrm{ED}$ approach is particularly suitable for our $\mathrm{N} 3 / \mathrm{TiO}_{2} / \mathrm{Ag}$ composite model system, as only a modest number of $\mathrm{CPU}$ cycles are needed to treat the $\mathrm{N} 3$ dye optical response using the real-time time-dependent density functional theory (RTTDDFT $)^{20}$ while the FDTD calculation ${ }^{21}$ uses even fewer resources to describe the optical properties of the $\mathrm{TiO}_{2} / \mathrm{Ag}_{2} \mathrm{O}$ / Ag substrate.

The N3 dye is one of the most popular light harvesters in DSSCs because of its broad absorption band and remarkable chemical stability. ${ }^{22}$ Structurally, an N3 dye molecule has two thiocyanate and two 4,4'-dicarboxy-2,2'-bipyridine functional groups all coordinated to a central ruthenium atom in the cisconfiguration (Figure 1). Interestingly, it was found by a recent first-principles study ${ }^{23}$ that the relative orientation of the bound

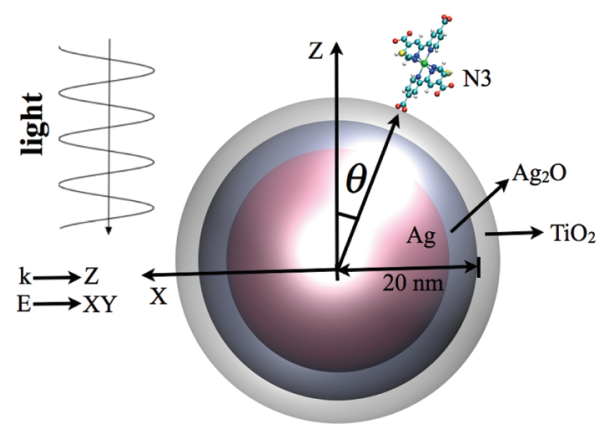

Figure 1. Structure of the $\mathrm{N} 3 / \mathrm{TiO}_{2} / \mathrm{Ag}$ composite DSSC, where the incident light propagates along axis $\mathrm{Z}$, and the polarization direction is uniformly distributed in the $\mathrm{XY}$ plane. The relative location of N3 dye on the $\mathrm{TiO}_{2}$ surface is indicated by the polar angle, $\theta$.

$\mathrm{N} 3$ dye on a $\mathrm{TiO}_{2}$ substrate is strongly influenced by the degree of hydration of the surface. On a perfectly clean $\mathrm{TiO}_{2}$ surface, each of the two 4,4'-dicarboxy-2,2'-bipyridine groups form two chemical bonds with titanium atoms, leading to an adsorption energy of $E_{\mathrm{ads}}=3.46 \mathrm{eV}$. In contrast, on a largely hydrated $\mathrm{TiO}_{2}$ substrate, the N3 dye adopts an orientation such that only one of its four carboxyl groups points toward the surface and the binding bipyridine group is parallel to the surface normal, reducing $E_{\text {ads }}$ to $1.56 \mathrm{eV}$. In terms of photoinjection efficiency, the amorphous $\mathrm{TiO}_{2}$ is known to outperform its naturally occurring crystalline form (e.g., anatase and rutile) due to its high-area photoanode ${ }^{24}$ that not only allows sequential cosensitization but also retards charge recombination. ${ }^{25}$ Therefore, it is not surprising that the mesoporous $\mathrm{TiO}_{2}$ film has become a de facto standard component of DSSCs by preventing electron injection back to the electrolytes. ${ }^{26}$

As a self-limiting process, atomic layer deposition (ALD) can be employed to fabricate thin films whose thickness is precisely controllable through the number of growth cycles. ${ }^{27}$ Moreover, the large variety of possible ALD precursors available allows for the deposition of many metal oxides, including $\mathrm{TiO}_{2}$, on silver nanoparticles. However, in the presence of the corrosive $\mathrm{I}^{-} / \mathrm{I}_{3}{ }^{-}$ solution, there is a minimum thickness of $2.0 \mathrm{~nm}$ of $\mathrm{TiO}_{2}$ required to prevent direct contact between the electrolyte and silver nanoparticle. ${ }^{28}$ This indicates that the $\mathrm{N} 3 / \mathrm{TiO}_{2} / \mathrm{Ag}$ composite DSSC requires careful structural control for optimum photovoltaic performance.

The remainder of the paper is organized as follows. In Section 2, a few details of the P-DSSC experiments are described that are important to the theoretical analysis, along with technical details of our modeling of the $\mathrm{N} 3 / \mathrm{TiO}_{2} / \mathrm{Ag}_{2} \mathrm{O}$ / $\mathrm{Ag}$ system within the framework of QM/ED. In Section 3, the computed absorption cross sections for the N3 dye with different interfacial architectures for the composite P-DSSC are presented along with corresponding IPCE enhancement factors. Finally, a comparison is made for the extinction spectrum and photocurrent profile between simulations and experiments, and some possible applications of QM/ED to DSSCs, in combination with other theoretical approaches, are briefly discussed.

\section{COMPUTATIONAL AND EXPERIMENTAL DETAILS}

2.1. Synthesis and Characterization of the Plasmonic DSSC. Silver NPs were synthesized according to a literature procedure. ${ }^{29}$ Briefly, $0.2 \mathrm{~g}$ of silver oxide was added to $200 \mathrm{~mL}$ of ultrapure water. The solution was heated to $70{ }^{\circ} \mathrm{C}$. The 
reaction proceeded for $4 \mathrm{~h}$ under a hydrogen atmosphere. Fluorine-doped tin oxide (FTO) was sonicated for $10 \mathrm{~min}$ each in soapy water, ethanol, and acetone. The slides were rinsed with isopropanol and dried under flowing nitrogen. The slides were soaked for $3 \mathrm{~h}$ in a 2 wt \% solution of 4-(polyvinyl)pyridine $(2 \mathrm{wt} \%)$ in ethanol. The slides were heated for $1 \mathrm{~h}$ at $110{ }^{\circ} \mathrm{C}$ and then were soaked in the colloidal silver solution for $24 \mathrm{~h}$.

Amorphous $\mathrm{TiO}_{2}$ was deposited from a titanium isopropoxide (TIP) precursor using a Cambridge Nanotech Savannah 100 ALD system. The chamber temperature and the TIP precursor were held at 200 and $80{ }^{\circ} \mathrm{C}$, respectively. The water was at room temperature, and the $\mathrm{N}_{2}$ flow rate was $20 \mathrm{sccm}$. The precursors were pulsed for $0.1 \mathrm{~s}$ each and held in the chamber for $1 \mathrm{~s}$ followed by a $12 \mathrm{~s}$ pump. Clean silicon chips were coated concurrently with the samples to monitor $\mathrm{TiO}_{2}$ film growth. Slides were soaked in a $0.5 \mathrm{mM}$ solution of N3 dye in ethanol for $24 \mathrm{~h}$.

The thicknesses were measured with a J.A. Woolam Co. M2000 variable angle spectroscopic ellipsometer. All thicknesses were relative to an uncoated wafer. The extinction spectra of the samples were measured with a Cary 5000 UVvis-NIR spectrometer. A Hitachi S-4800 SEM was used with a $10.0 \mathrm{kV}$ accelerating voltage to obtain micrographs for Ag NP size characterization.

2.2. Architecture of the $\mathrm{N} 3 / \mathrm{TiO}_{2} / \mathrm{Ag}$ Composite DSSC. The solar cell consists of core-shell nanoparticles (schematically pictured in Figure 1) that are deposited in an electrochemical cell with FTO electrodes and an $\mathrm{I}^{-} / \mathrm{I}_{3}{ }^{-}$ electrolyte as previously described by Standridge et al. ${ }^{10}$ Since neither the $\mathrm{I}^{-} / \mathrm{I}_{3}^{-}$electrolyte solution nor the working electrode is photoactive, they are not included in our theoretical modeling. As shown in Figure 1, the N3 dye is adsorbed on the surface of the $\mathrm{TiO}_{2}$ layer. Between $\mathrm{TiO}_{2}$ and the $\mathrm{Ag}$ nanoparticle core, there is an intermediate layer of $\mathrm{Ag}_{2} \mathrm{O}^{30}$ This $\mathrm{Ag}_{2} \mathrm{O}$ layer is an oxidation byproduct of $\mathrm{ALD}$ under ambient conditions and can be diminished by high-vacuum sputtering. ${ }^{27}$ Although the diameter of the oxidized Ag core was determined to be $36 \pm 3 \mathrm{~nm}$ by scanning electron microscopy $(\mathrm{SEM})^{10}$ as shown in the Supporting Information, the thickness of the $\mathrm{Ag}_{2} \mathrm{O}$ layer, $\mathrm{D}_{\mathrm{Ag}_{2} \mathrm{O}}$, is not yet known and will be estimated by our FDTD calculations.

Given the typical $1 \mathrm{~nm}$ uncertainty in SEM measurements, $R_{\mathrm{Ag}}+D_{\mathrm{Ag}_{2} \mathrm{O}}$ is taken to be $20 \mathrm{~nm}$ as an upper limit to the oxidized $\mathrm{Ag}$ particle size, where $R_{\mathrm{Ag}}$ is the radius of the pure $\mathrm{Ag}$ core. At the same time, the thickness of the $\mathrm{TiO}_{2}$ layer, $D_{\mathrm{TiO}_{2}}$, has been accurately measured by variable angle spectroscopic ellipsometer relative to an uncoated silicon wafer. In the present study, three values of $D_{\mathrm{TiO}_{2}}$ were investigated: 2.0, 4.2, and $8.3 \mathrm{~nm}$.

In the absence of experimental evidence for preferred binding sites of the N3 dye on the surface, the N3 is assumed to be homogeneously distributed on the $\mathrm{TiO}_{2}$ surface with a polar angle $\theta$ (see Figure 1 ) that is equally spaced by $15^{\circ}$ from $\theta=0^{\circ}$ to $\theta=180^{\circ}$. Also, the $\mathrm{N} 3$ is assumed bound through one carboxylate group, and azumuthal effects are dealt with by polarization averaging. To mimic direct solar radiation perpendicular to the irradiated surface, the incident light is taken to propagate along the $\mathrm{Z}$ axis in Figure 1 with its polarization direction equally distributed in the $\mathrm{XY}$ plane. Please note that there is only one N3 molecule in our model system, given its low surface concentration on the easily hydrated $\mathrm{TiO}_{2}$ surface. ${ }^{23,31}$

2.3. Estimation of the Thickness of $\mathrm{Ag}_{2} \mathrm{O}, \mathrm{D}_{\mathrm{Ag}_{2} \mathrm{O}}$. Unlike the $\mathrm{Ag}$ core, the intermediate $\mathrm{Ag}_{2} \mathrm{O}$ layer is a semiconducting material that does not exhibit any plasmonic features. However, its presence and thickness influences the magnitude of the surface electric fields, which in turn determine the IPCE of the DSSC. Although $D_{\mathrm{Ag}_{2} \mathrm{O}}$ for a $\mathrm{Ag} / \mathrm{Ag}_{2} \mathrm{O}$ layered system can be conveniently measured by the angle-resolved reflectivity for swave and p-wave polarization, ${ }^{32}$ its reliability in the range $0 \mathrm{~nm}$ $<D_{\mathrm{Ag}_{2} \mathrm{O}}<5 \mathrm{~nm}$ is uncertain due to a sharp red shift of the plasmon resonance for small oxide layer thicknesses. Therefore, for our $\mathrm{Ag} / \mathrm{Ag}_{2} \mathrm{O} / \mathrm{TiO}_{2}$ nanoparticle structures with a very thin $\mathrm{Ag}_{2} \mathrm{O}$ layer, the angle-resolved reflectivity would not be useful. An alternative protocol to estimate $D_{\mathrm{Ag}_{2} \mathrm{O}}$ is to compare the experimentally obtained plasmon wavelength for the $\mathrm{Ag} / \mathrm{Ag}_{2} \mathrm{O}$ / $\mathrm{TiO}_{2}$ nanoparticle with the simulated extinction profile maximum; optical theory has been very successful at interpreting and predicting such plasmonic phenomena for structures like this. ${ }^{33}$ The MEEP FDTD simulation package ${ }^{34}$ was used to calculate the transmission loss of a delayed Gaussian electric pulse after it had passed through the composite DSSC. In our FDTD calculations, the delay time and the peak width of the electric pulse were carefully chosen to centralize the optical response within the visible region of interest. In addition, $D_{\mathrm{TiO}_{2}}$ was fixed at $2.0 \mathrm{~nm}$, and $D_{\mathrm{Ag}_{2} \mathrm{O}}$ was varied from 1.0 to $9.0 \mathrm{~nm}$ to examine the red shift of plasmon excitation. The dielectric functions, $\varepsilon$, for $\mathrm{Ag}$ and $\mathrm{Ag}_{2} \mathrm{O}$ were taken from Johnson and Christy ${ }^{35}$ and Garcia-Macedo, ${ }^{36}$ respectively, while our experimentally measured values for the amorphous $\mathrm{TiO}_{2}$ are shown in Figure 2 as a function of

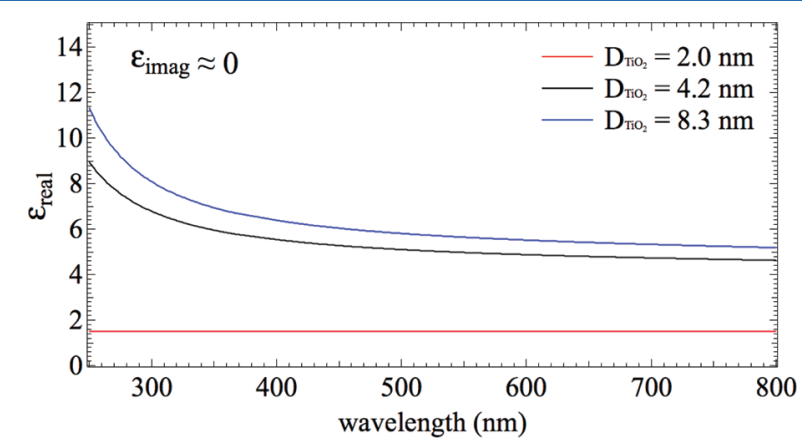

Figure 2. Dependence of the $\mathrm{TiO}_{2}$ layer's dielectric function on its thickness.

thickness. This shows that the real part of $\varepsilon$ decreases rapidly with decreasing $D_{\mathrm{TiO}_{2}}$ below $4.2 \mathrm{~nm}$, eventually resulting in a frequency-invariant $\varepsilon$ at $\sim 1.6$ for $D_{\mathrm{TiO}_{2}}=2.0 \mathrm{~nm}$. After obtaining the extinction spectrum through Fourier transformation of the time-dependent transmission loss, the optimal $D_{\mathrm{Ag}_{2} \mathrm{O}}$ is the one yielding an extinction maximum closest to the reported wavelength of $460 \mathrm{~nm} .{ }^{10}$ We have also performed extended Mie theory ${ }^{37}$ calculations for the same core/shell structures to verify the quality of the FDTD calculations and further explore optical properties.

2.4. QM/ED Calculation of the Plasmon-Enhanced Absorption Cross Sections of N3. A key parameter in QM/ ED simulations is the scattering response function $\lambda$, which measures the enhancement in the local electric field due to 
plasmon excitation. ${ }^{19}$ The $\lambda$ value for a given binding site of the $\mathrm{N} 3$ dye, $r$, was evaluated from the ratio of the scattered electric field $E_{\text {sca }}(r, \omega)$ and the incident field $E_{0}(r, \omega)$ using FDTD calculations. Subsequently, the total electric field $E_{0}(r, \omega)+$ $E_{\text {sca }}(r, \omega)$ was added to the $\mathrm{N} 3$ dye Hamiltonian to drive the propagation of its wave function according to the timedependent Schrödinger equation. ${ }^{38}$ After Fourier transformation of the induced dipole moment followed by normalization by the incident field, the linear molecular polarizability, $\alpha(\omega)$, over a wide frequency range is determined in a single RTTDDFT simulation. To include for spatial averaging over the incident polarization directions, the absorption cross section, $\sigma_{\mathrm{abs}}(\omega)$, for the bound N3 dye irradiated by light with a fixed propagation direction is given by the following:

$$
\begin{aligned}
\sigma(\omega)= & \frac{4 \pi \omega}{c} \\
& \left\langle\frac { 1 } { 2 } \left(\alpha_{i i}\left(1+\lambda^{*}{ }_{i i}\right)+\alpha_{j j}\left(1+\lambda^{*}{ }_{j j}\right)+\alpha_{i j} \lambda^{*}{ }_{i j}+\alpha_{j i} \lambda\right.\right. \\
& \left.\left.*_{j i}+\alpha_{i k} \lambda^{*_{i k}}+\alpha_{j k} \lambda^{*}{ }_{j k}\right)\right\rangle_{\text {imag }}
\end{aligned}
$$

where \langle\rangle$_{\text {imag }}$ denotes the imaginary part and $*$ indicates the complex conjugate operator. Please refer to the Supporting Information for a detailed derivation of eq 1 .

The CP2K molecular simulation package ${ }^{39}$ was utilized to perform the RT-TDDFT calculations with the GoedeckerTeter-Hutter (GTH) dual-space Gaussian pseudopotential, ${ }^{40}$ the Perdew-Burke-Ernzerhof (PBE) exchange-correlationfunctional $^{41}$ and the polarized valence-double- $\zeta$ (PVDZ) basis set. $^{42}$ It is also found that a total simulation time of $50 \mathrm{fs}$ is sufficiently long to converge $\sigma(\omega)$ in the visible region if a commonly used value of $0.1 \mathrm{eV}$ is chosen for the damping factor, $\Gamma$, to empirically reflect the effect of quantum dephasing and vibronic coupling.

2.5. Spatial Averages of $\sigma(\omega)$ and the IPCE Enhancement Factor, $\boldsymbol{\eta}$. Under the assumption of a uniform distribution of $\mathrm{N} 3$ dye locations on the $\mathrm{TiO}_{2}$ surface, the spatial average of its absorption cross section, $\overline{\sigma(\omega)}$, is given by the following:

$$
\overline{\sigma(\omega)}=\int_{0}^{\pi / 2} d \theta \sigma(\omega, \theta) \sin \theta
$$

where $\sin \theta$ is the usual polar angle weighting factor. Here, the upper limit of the integration in eq 2 is reduced from $180^{\circ}$ to $90^{\circ}$ due to dipolar symmetry of the field for the small particle we consider. Due to the weak solar radiation intensity, light absorption is usually considered as a significant limiting step for a photovoltaic device. Therefore, the IPCE is approximately proportional to $\overline{\sigma(\omega)}$, which is further weighted from 280 to $900 \mathrm{~nm}$ by the AM1.5G solar radiation spectrum as shown in the Supporting Information, ${ }^{43} \operatorname{SRS}(\omega)$ :

$$
\operatorname{IPCE} \propto \int \operatorname{SRS}(\omega) \overline{\sigma(\omega)} d \omega
$$

In the present study, the IPCE associated with bare N3 is chosen as our reference system. Accordingly, the IPCE enhancement factor, $\eta$, is defined as follows:

$$
\eta=\frac{\mathrm{IPCE}}{\mathrm{IPCE}^{0}}
$$

where $\mathrm{IPCE}^{0}$ is given by eq 3 but for bare N3 dye in vacuum.

\section{RESULTS}

The simulated and experimental profiles of the bare N3 dye, $\sigma^{0}(\omega)$, are shown in Figure 3. Note that the dimensionless

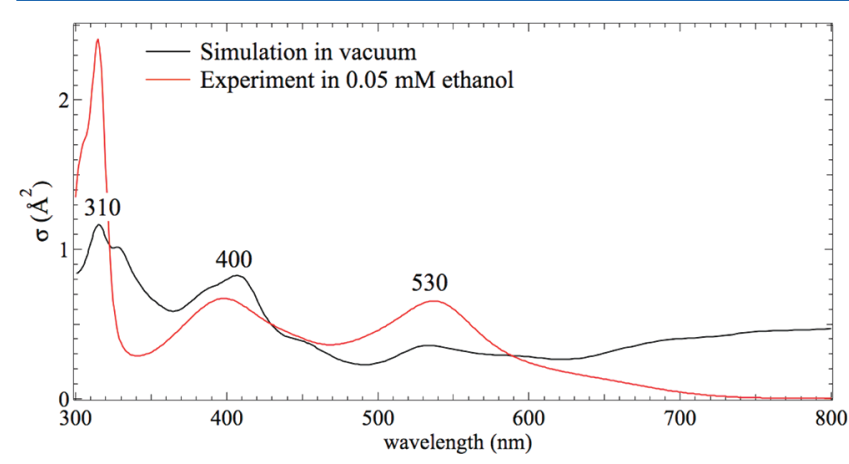

Figure 3. Absorption cross section of the N3 dye, $\sigma^{0}(\omega)$, simulated in vacuum (black) and measured in $0.05 \mathrm{mM}$ ethanol solutions (red), respectively.

experimental profile has been rescaled to be comparable with the simulated one. The figure shows three absorption peaks, at 310,400 , and $530 \mathrm{~nm}$. Although the height of the primary peak at $310 \mathrm{~nm}$ is significantly higher in the measured spectrum, the average behavior of the calculated RT-TDDFT results is in good agreement with experiment. Solvation effects in ethanol are likely an important factor why the results are not in better agreement, as this usually selectively stabilizes excited states with large charge separation, or even enlarges the corresponding transition dipole moments. ${ }^{44}$ Nevertheless, the $\mathrm{N} 3$ dye reveals itself as a broad-band light harvester that is critical for photovoltaic applications.

To study the effect of a $\mathrm{Ag}_{2} \mathrm{O}$ layer on the $\mathrm{TiO}_{2}$-coated $\mathrm{Ag} /$ $\mathrm{Ag}_{2} \mathrm{O}$ particle, in Figure 4, we plot the plasmon wavelength as a

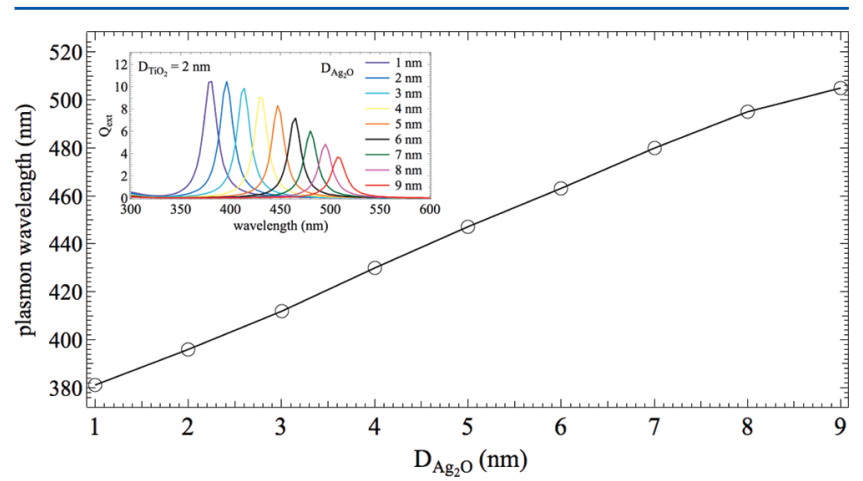

Figure 4. Dependence of plasmon wavelength on the thickness of $\mathrm{Ag}_{2} \mathrm{O}\left(D_{\mathrm{Ag}_{2} \mathrm{O}}\right)$ when the thickness of $\mathrm{TiO}_{2}\left(D_{\mathrm{TiO}_{2}}\right)$ is fixed at $2.0 \mathrm{~nm}$, and the radius of the oxidized silver sphere $\left(R_{\mathrm{Ag}}+D_{\mathrm{Ag}_{2} \mathrm{O}}\right)$ is fixed at $20.0 \mathrm{~nm}$. The results from FDTD simulation and Mie theory are presented in red and black lines, respectively. In addition, the extinction profile as a function of $D_{\mathrm{Ag}_{2} \mathrm{O}}$, which is calculated from Mie theory, is illustrated in the inset.

function of $\mathrm{Ag}_{2} \mathrm{O}$ thickness $\mathrm{D}_{\mathrm{Ag}_{2} \mathrm{O}}$, for fixed $\mathrm{TiO}_{2}$ thickness of 2 nm. Both FDTD and extended Mie theory were used for this evaluation, and we see that they are in excellent agreement (which shows the high quality of the FDTD results). Also, the inset shows the calculated Mie theory extinction profiles for the $\mathrm{TiO}_{2} / \mathrm{Ag}_{2} \mathrm{O} / \mathrm{Ag}$ particle for various thicknesses of $\mathrm{Ag}_{2} \mathrm{O}$ in the 
range 1-9 nm. Further technical details of the Mie theory calculation are provided in the Supporting Information. Figure 4 shows that the plasmon excitation red shifts and decreases in intensity with increasing layer thickness. The red shifting as the $\mathrm{Ag}_{2} \mathrm{O}$ layer thickness increases is the expected result given the large real part of the $\mathrm{Ag}_{2} \mathrm{O}$ dielectric function. The damping is due to absorption by the $\mathrm{Ag}_{2} \mathrm{O}$ and reflects the importance of the imaginary part of the dielectric constant. In comparison to the experimental extinction profile, ${ }^{10}$ the computed result with $D_{\mathrm{Ag}_{2} \mathrm{O}}=6 \mathrm{~nm}$ exhibits the most consistent plasmon wavelength. Therefore, $6 \mathrm{~nm}$ is regarded as the correct value for $D_{\mathrm{Ag}_{2} \mathrm{O}}$ and will be applied in the rest of our simulations.

The angle-resolved $\sigma(\omega)$ shown in Figure $5 \mathrm{a}$ has been calculated by the QM/ED approach for the $\mathrm{N} 3 / \mathrm{TiO}_{2} / \mathrm{Ag}_{2} \mathrm{O} /$

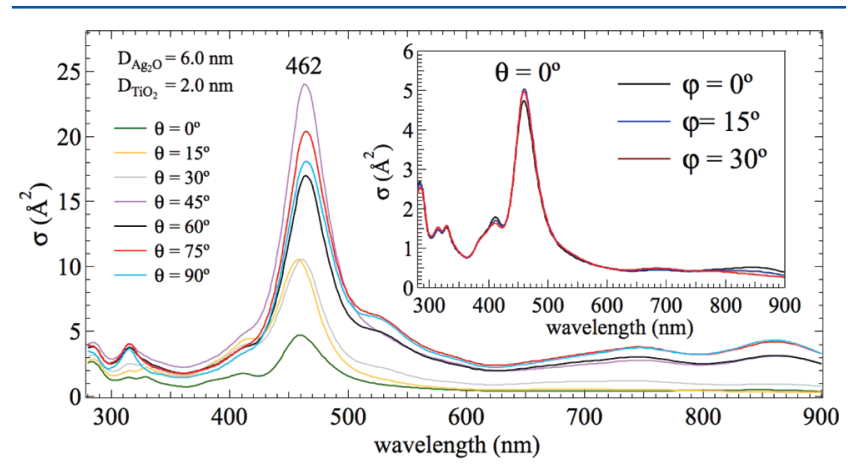

(a)

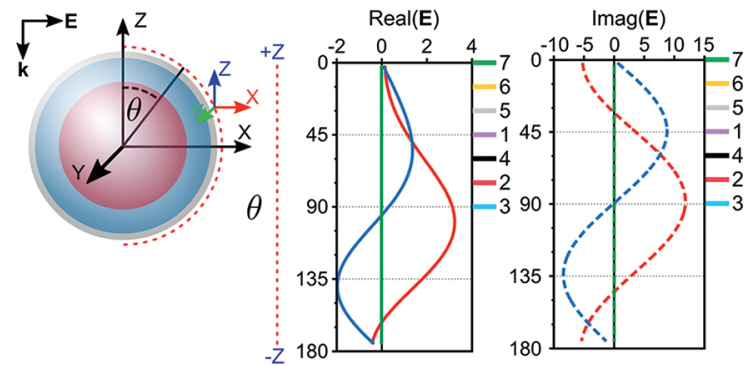

(b)

Figure 5. (a) Absorption cross section of the N3 dye, $\sigma(\omega)$, as a function of the polar angle, $\theta$, for a composite substrate with $D_{\mathrm{TiO}_{2}}=$ $2.0 \mathrm{~nm}$ and $D_{\mathrm{Ag}_{2} \mathrm{O}}=6.0 \mathrm{~nm}$. The inset shows the variance of $\sigma(\omega)$ at $\theta$ $=0^{\circ}$ when the angle, $\varphi$, between the $\mathrm{N} 3$ dye's binding pyridine plane and the $\mathrm{TiO}_{2}$ surface normal changes from $0^{\circ}$ to $30^{\circ}$. (b) A breakdown of the Cartesian electric field components in the $x z$ plane. The electric field was calculated along the dashed red line in the $x z$ plane (left panel). The real (center panel) and imaginary (right panel) parts of the field are plotted as a function of $\theta$. Red, green, and blue lines correspond to the $x, y$ and $z$ components of the field, respectively. The colors of the numbered labels on the top right of the panels correspond to the curves in part (a) of this figure. The numbers themselves represent the heights of the peaks from 1 (the strongest dye absorption), to 7 (the weakest absorption).

Ag system. As anticipated, the light absorption is significantly enhanced at the plasmon wavelength of $462 \mathrm{~nm}$ for most positions of the $\mathrm{N} 3$ dye on the nanoparticle surface, illustrating strong coupling between the substrate's plasmonic scattered field and the dye's transition dipole moment. When $\theta=45^{\circ}$, $\sigma(\omega)$ reaches its maximum of $\sim 25 \AA^{2}$ at $\lambda=463 \mathrm{~nm}$, while for $\theta$ $=0^{\circ}, \sigma(\lambda=462 \mathrm{~nm})$ drops to $\sim 5 \AA^{2}$, approximately a 5-fold reduction. The notable variation of $\sigma(\omega)$ with $\theta$ can be primarily explained by two factors: the first one is the relative electric field enhancement factor at the location of the N3 dye; the second one is the relative direction of the plasmonic scattered field at the position of the molecule and the molecular transition dipole moment. To illustrate these points, Figure 6

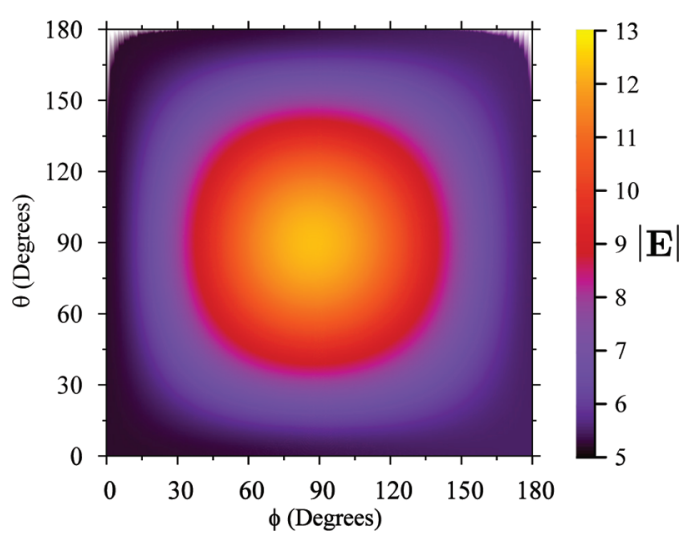

Figure 6. Electric field strength at the resonance wavelength of 463 $\mathrm{nm}$ on the surface of a $14 \mathrm{~nm}$ radius silver sphere with a $6 \mathrm{~nm} \mathrm{Ag}_{2} \mathrm{O}$ coating and a $2 \mathrm{~nm} \mathrm{TiO}_{2}$ capping layer. Only half of the sphere is shown, and the field is polarized in the $\mathrm{x}$ direction. The electric field has been normalized to the incident field.

shows the electric field strength $0.5 \mathrm{~nm}$ away from the $\mathrm{TiO}_{2}$ surface as a function of $\theta$ and the azimuthal angle $\varphi$ for the case of X-polarized radiation. This shows the expected dipole-field pattern, with a peak electric field strength along the $\mathrm{X}$ axis that is 13 times the incident field strength. In the Supporting Information, we investigate the noninteracting case and show that the relative orientation of the dye polarizability and the electric field cause the maximum dye absorption cross section to be at an angle $\theta<90^{\circ}$. In both interacting and noninteracting cases, there is a significant $\varphi$-dependence to the field, but the average over polarizations suppresses this dependence.

Note that there is only a small change in $\sigma(\omega)$ at $\theta=0^{\circ}$ when the angle between the N3 dye's binding pyridine plane and the $\mathrm{TiO}_{2}$ surface normal is varied from $0^{\circ}$ to $30^{\circ}$ (inset of Figure 5a). This shows that the light absorption efficiency of the N3 dye is not very sensitive to its relative orientation with respect to the $\mathrm{TiO}_{2}$ surface.

Although the primary enhancement occurs near the plasmon wavelength, $\sigma(\omega)$ is also moderately enhanced in the wavelength range from 600 to $900 \mathrm{~nm}$, further aiding the collection of solar energy as long as electron injection still works at these wavelengths. As shown in the solar radiation spectrum (see Supporting Information), the solar radiation intensity has a much slower decay for the red side than on the blue side, suggesting the importance of infrared absorption. Given the bare N3 dye's already impressive performance for shorter-wavelength absorption (Figure 3), the fact that the plasmonic substrate actually facilitates the bound N3 dye to achieve a broader and more balanced absorption profile (excluding the strongly amplified region near $450 \mathrm{~nm}$ ) makes this structure very appropriate for an efficient DSSC.

If the N3 dye is assumed to have no preference in its binding site on the $\mathrm{TiO}_{2}$ surface, the computed profiles of $\overline{\sigma(\omega)}$ for different $D_{\mathrm{TiO}_{2}}$ are shown in Figure 7. This shows that our computed $\overline{\sigma(\omega)}$ profiles are directly comparable to the experimental extinction difference spectrum. ${ }^{10}$ When $D_{\mathrm{TiO}_{2}}$ 


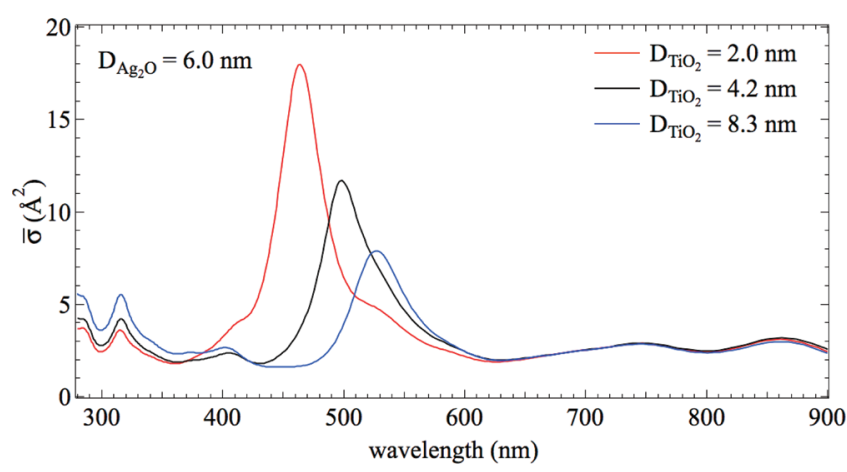

Figure 7. Spatially averaged absorption cross section of the N3 dye, $\sigma(\omega)$, for different thicknesses of the $\mathrm{TiO}_{2}$ layer, $D_{\mathrm{TiO} 2}$.

increases from 2.0 to $4.2 \mathrm{~nm}$, a red shift of the extinction peak by $40 \mathrm{~nm}$ is observed in our simulations in addition to a 40\% reduction in peak height. These observations are consistent with experimental findings of a $50 \mathrm{~nm}$ red shift and $75 \%$ height reduction. ${ }^{10}$ This diminished plasmon enhancement is due to the increased separation between the $\mathrm{N} 3$ dye and Ag core. With a further increase of $D_{\mathrm{TiO}_{2}}$ from 4.2 to $8.3 \mathrm{~nm}$, the extinction peak shift is considerably diminished, reflecting the finite range of the dielectric response of the plasmon. Although there is no experimental extinction data for $D_{\mathrm{TiO}_{2}}$ beyond $4.8 \mathrm{~nm}$, the trend showing a smaller peak shift can be also inferred from the nearly invariant extinction peak when $D_{\mathrm{TiO}_{2}}$ changes from 4.2 to $4.8 \mathrm{~nm}$ in the experiments. ${ }^{10}$ In addition, the computed extinction peak for $D_{\mathrm{TiO}_{2}}=8.3 \mathrm{~nm}$ is only slightly lower than that for $D_{\mathrm{TiO}_{2}}=4.2 \mathrm{~nm}$ even with a much larger separation between $\mathrm{Ag}$ and $\mathrm{N} 3$. The reduction in loss of plasmon enhancement for the thicker dielectric $\mathrm{TiO}_{2}$ layer is also reflected in the computed $\eta$ (Table 1), where the IPCE enhancement factors for $D_{\mathrm{TiO}_{2}}=4.2 \mathrm{~nm}$ and $D_{\mathrm{TiO}_{2}}=8.3 \mathrm{~nm}$ only differ by $15 \%$.

Table 1. Dependence of the IPCE Enhancement Factor, $\boldsymbol{\eta}$, on $\mathrm{D}_{\mathrm{TiO}_{2}}$

\begin{tabular}{cc}
$D_{\mathrm{TiO}_{2}}(\mathrm{~nm})$ & $\eta$ \\
2.0 & 9.9 \\
4.2 & 8.1 \\
8.3 & 6.9 \\
\hline
\end{tabular}

\section{DISCUSSION}

Our calculated estimate of the plasmon enhancement for the P-DSSC cell studied by Standridge et al. ${ }^{10}$ is $\eta=9.9$. This refers to $D_{\mathrm{TiO}_{2}}=2.0 \mathrm{~nm}$ and $D_{\mathrm{Ag}_{2} \mathrm{O}}=6 \mathrm{~nm}$, which are consistent with the ALD deposition used, and the observed extinction maximum for the cell that gave the highest efficiency. This efficiency can be compared to the value 6.0 from the experimental photocurrent measurement, ${ }^{10}$ although it should be noted that the actual enhancement measured was 8.4 . The value 6.0 was derived from the 8.4 value by dividing by a 1.4 factor that was the estimated increase in surface area in the PDSSC cell compared to the comparable DSSC due to the presence of dye-coated silver particles. Of course, there is uncertainty in either of these numbers particularly due to the experimentally unresolved thickness of $\mathrm{Ag}_{2} \mathrm{O}$. For example, our calculated $\eta$ of 6.9 for $D_{\mathrm{TiO}_{2}}=8.3 \mathrm{~nm}$ seems even closer to the reported value of 6.0 than the other two geometries despite a significant $50 \mathrm{~nm}$ red shift of the absorption peak compared to experiments. But overall, we see that the agreement is reasonable, which indicates that the hybrid QM/ED method is useful for describing a cell where photocurrent enhancement is proportional to absorption enhancement. It should be also noted the usually faster charge recombination rate for thinner $\mathrm{TiO}_{2}$ layers may become an undesired process competing with the electron injection, resulting in reduced photocurrent.

Precise control over a nanoparticle's size, shape, and composition is now possible with advanced nanofabrication techniques, such as dip-pen nanolithography, ${ }^{45}$ photodriven nanoprism synthesis, ${ }^{46}$ and directed self-assembly. ${ }^{47}$ More recently, programmable two-dimensional patterns have been achieved by solvent-assisted embossing, ${ }^{48}$ leading to pronounced subradiant plasmon modes with longer lifetime and tunable resonance wavelength. ${ }^{49}$ Given the already abundant choices of semiconductor films and organic dye molecules, we can easily construct a P-DSSC with a desired combination of the light concentrator, charge carrier, and solar harvester. In the present applications, these respectively correspond to the $\mathrm{Ag}$ core, $\mathrm{TiO}_{2}$ layer, and $\mathrm{N} 3$ dye. The greatest challenge we currently face for the computational modeling of P-DSSC is how to determine nanoarchitectures, which optimize the IPCE enhancement factor. Given the almost-infinite variations possible for assembling the key components of a P-DSSC, in silico virtual screening of nanostructures is a valuable activity. On the other hand, we also need to ensure accuracy of the modeling because the simulated results are very sensitive to structural parameters.

As demonstrated by the comparison between our simulation results and experimental findings, the hybrid QM/ED approach achieves a reasonable compromise between the competing requirements of accuracy and efficiency. Consequently, the QM/ED simulation is believed to provide a practical tool for the systematic design of P-DSSC. Perhaps, the largest uncertainty in using QM/ED to predict the IPCE enhancement factor stems from ignorance of the electron injection process, which occurs much faster than the light absorption under weak irradiance. However, it is completely plausible to combine QM/ ED with our newly developed constrained real-time timedependent density functional theory (CRT-TDDFT) $)^{50}$ to explicitly account for the electron injection that is thought to result from light absorption. In this sense, a seamless integration between QM/ED and CRT-TDDFT is expected to provide a more complete picture of photoinduced electron transfer in P-DSSC.

\section{ASSOCIATED CONTENT}

\section{S Supporting Information}

Derivation of the spatially averaged dye absorption cross section, Mie theory, dependence of the plasmon wavelength on oxide thickness, comparison of experimental silver oxide dielectric functions, the AM1.5G solar spectrum, and SEM characterization of Ag NP on FTO substrate before ALD. This material is available free of charge via the Internet at http:// pubs.acs.org. 


\section{AUTHOR INFORMATION}

\section{Corresponding Author}

*Fax: 847-491-7713; Phone: 847-491-5657; E-mail: schatz@ chem.northwestern.edu.

\section{Notes}

The authors declare no competing financial interest.

\section{ACKNOWLEDGMENTS}

The research was supported by the ANSER Energy Frontier Research Center Grant DE-SC0001059, funded by the U.S. Department of Energy, Office of Science, and Office of Basic Energy Sciences. The computational resources utilized in this research were provided by Shanghai Supercomputer Center.

\section{REFERENCES}

(1) Barnham, K. W. J.; Mazzer, M.; Clive, B. Nat. Mater. 2006, 5, $161-164$.

(2) Kamat, P. V. J. Phys. Chem. C 2007, 111, 2834-2860.

(3) Miles, R. W.; Hynes, K. M.; Forbes, I. Prog. Cryst. Growth Charact. Mater. 2005, 51, 1-42.

(4) Grätzel, M. J. Photochem. Photobiol., C 2003, 4, 145-153.

(5) Rehm, J. M.; McLendon, G. L.; Nagasawa, Y.; Yoshihara, K.; Moser, J.; Grätzel, M. J. Phys. Chem. 1996, 100, 9577-9578.

(6) Berginc, M.; Opara Krasovec, U.; Jankovec, M.; Topic, M. Sol. Energy Mater. Sol. Cells 2007, 91, 821-828.

(7) O’Regan, B.; Gratzel, M. Nature 1991, 353, 737-740.

(8) Green, M. A.; Emery, K.; Hishikawa, Y.; Warta, W. Prog. Photovoltaics 2010, 18, 346-352.

(9) Hagglund, C.; Zach, M.; Kasemo, B. Appl. Phys. Lett. 2008, 92, 013113-3.

(10) Standridge, S. D.; Schatz, G. C.; Hupp, J. T. J. Am. Chem. Soc. 2009, 131, 8407-8409.

(11) Brown, M. D.; Suteewong, T.; Kumar, R. S. S.; D’Innocenzo, V.; Petrozza, A.; Lee, M. M.; Wiesner, U.; Snaith, H. J. Nano Lett. 2010, 11, 438-445.

(12) Sarkar, S.; Makhal, A.; Bora, T.; Baruah, S.; Dutta, J.; Pal, S. K. Phys. Chem. Chem. Phys. 2011, 13, 12488-12496.

(13) Ferry, V. E.; Munday, J. N.; Atwater, H. A. Adv. Mater. 2010, 22, 4794-4808.

(14) Jeong, N. C.; Prasittichai, C.; Hupp, J. T. Langmuir 2011, 27, 14609-14614.

(15) Qi, J.; Dang, X.; Hammond, P. T.; Belchers, A. M. ACS Nano 2011, 5, 7108-7116.

(16) Brown, M. D.; Suteewong, T.; Kumar, R. S. S.; D’Innocenzo, V.; Petrozza, A.; Lee, M. M.; Wiesner, U.; Snaith, H. J. Nano Lett. 2011, $11,438-445$.

(17) McMahon, J. M.; Gray, S. K.; Schatz, G. C. Phys. Rev. Lett. 2009, 103, 097403-097404.

(18) McMahon, J. M.; Gray, S. K.; Schatz, G. C. Phys. Rev. B 2010, $82,035423$.

(19) Chen, H.; McMahon, J. M.; Ratner, M. A.; Schatz, G. C. J. Phys. Chem. C 2010, 114, 14384-14392.

(20) Yabana, K.; Bertsch, G. F. Phys. Rev. B 1996, 54, 4484.

(21) Kane, Y. IEEE Trans. Antennas Propag. 1966, 14, 302-307.

(22) Nazeeruddin, M. K.; Kay, A.; Rodicio, I.; Humphry-Baker, R.; Mueller, E.; Liska, P.; Vlachopoulos, N.; Graetzel, M. J. Am. Chem. Soc. 1993, 115, 6382-6390.

(23) Mäkinen, V.; Honkala, K.; Häkkinen, H. J. Phys. Chem. C 2011, $115,9250-9259$.

(24) Martinson, A. B. F.; Elam, J. W.; Liu, J.; Pellin, M. J.; Marks, T. J.; Hupp, J. T. Nano Lett. 2008, 8, 2862-2866.

(25) Shalom, M.; Albero, J.; Tachan, Z.; Martínez-Ferrero, E.; Zaban, A.; Palomares, E. J. Phys. Chem. Lett. 2010, 1, 1134-1138.

(26) Shalom, M.; Dor, S.; Rühle, S.; Grinis, L.; Zaban, A. J. Phys. Chem. C 2009, 113, 3895-3898.

(27) George, S. M. Chem. Rev. 2009, 110, 111-131.
(28) Standridge, S. D.; Schatz, G. C.; Hupp, J. T. Langmuir 2009, 25, 2596-2600.

(29) Evanoff, D. D.; Chumanov, G. J. Phys. Chem. B. 2004, 108, 13957-13962.

(30) Kuo, Y.-L.; Chen, H.-W.; Ku, Y. Thin Solid Films 2007, 515, 3461-3468.

(31) Mamontov, E.; Vlcek, L.; Wesolowski, D. J.; Cummings, P. T.; Rosenqvist, J.; Wang, W.; Cole, D. R.; Anovitz, L. M.; Gasparovic, G. Phys. Rev. E 2009, 79, 051504.

(32) Santillán, J. M. J.; Scaffardi, L. B.; Schinca, D. C.; Videla, F. A. J. Opt. 2010, 12, 045002 .

(33) Odom, T. W.; Schatz, G. C. Chem. Rev. 2011, 111, 3667-3668.

(34) Oskooi, A. F.; Roundy, D.; Ibanescu, M.; Bermel, P.; Joannopoulos, J. D.; Johnson, S. G. Comput. Phys. Commun. 2010, 181, 687-702.

(35) Johnson, P. B.; Christy, R. W. Phys. Rev. 1972, 6, 4370.

(36) Renteria-Tapia, V. M.; Valverde-Aguilar, G.; Garcia-Macedo, J. A. Plasmonics: Met. Nanostruct. Opt. Prop. V 2007, 6641, 66411W1W7.

(37) Morris, V. J.; Jennings, B. R. Biophys. J. 1977, 17, 95-101.

(38) Schrödinger, E. R. J. A. Phys. Rev. 1926, 28, 1049.

(39) VandeVondele, J.; Krack, M.; Mohamed, F.; Parrinello, M.; Chassaing, T.; Hutter, J. Comput. Phys. Commun. 2005, 167, 103-128.

(40) Goedecker, S.; Teter, M.; Hutter, J. Phys. Rev. B 1996, 54, 1703.

(41) Perdew, J. P.; Burke, K.; Ernzerhof, M. Phys. Rev. Lett. 1996, 77, 3865.

(42) Woon, D. E.; Dunning, J. T. H. J. Chem. Phys. 1994, 100, 29752988.

(43) Bird, R. E.; Hulstrom, R. L.; Lewis, L. J. Solar Energy 1983, 30, 563-573.

(44) Curutchet, C.; Scholes, G. D.; Mennucci, B.; Cammi, R. J. Phys. Chem. B 2007, 111, 13253-13265.

(45) Piner, R. D.; Zhu, J.; Xu, F.; Hong, S.; Mirkin, C. A. Science 1999, 283, 661-663.

(46) Jin, R.; Cao, Y.; Mirkin, C. A.; Kelly, K. L.; Schatz, G. C.; Zheng, J. G. Science 2001, 294, 1901-1903.

(47) Ozin, G. A.; Hou, K.; Lotsch, B. V.; Cademartiri, L.; Puzzo, D. P.; Scotognella, F.; Ghadimi, A.; Thomson, J. Mater. Today 2009, 12, $12-23$.

(48) Lee, M. H.; Huntington, M. D.; Zhou, W.; Yang, J.-C.; Odom, T. W. Nano Lett. 2010, 11, 311-315.

(49) Zhou, W.; Odom, T. W. Nat. Nano 2011, 6, 423-427.

(50) Chen, H.; Ratner, M. A.; Schatz, G. C. J. Phys. Chem. C 2011, 115, 18810-18821.

\section{NOTE ADDED AFTER ASAP PUBLICATION}

This paper was published to the Web on April 27, 2012, with an error to Figure 5's caption. The corrected version was reposted on May 2, 2012. 